\title{
Adiabatic Invariance for Tether Deployment/Retrieval Problem
}

\author{
Shi-Sheng Chu \\ Ph.D. \\ Institute of Applied Mechanics \\ National Taiwan University, Taipei \\ Taiwan, R.O.C. \\ sschu@tao.iam.ntu.edu.tw
}

\author{
Li-Sheng Wang \\ Professor \\ Institute of Applied Mechanics \\ National Taiwan University, Taipei \\ Taiwan, R.O.C. \\ wangli@tao.iam.ntu.edu.tw
}

\begin{abstract}
The idea of Tethered-Satellite-System (TSS) has been tested twice on the space shuttle in the past few years, however both failed. It is desirable to study the complicated dynamics of TSS more deeply. During the deployment phase or the retrieval phase of TSS operation, the length of the tether is varied slowly. This process may lead to a suitable application of the theory of adiabatic invariance. In short, adiabatic (or asymptotic) invariance is a quantity that is almost invariant when some parameter of the physical system is slowly changed. In this paper, we compute an adiabatic invariance for an approximate model of TSS during deployment/retrieval mode. It is observed that as the length of the tether becomes larger slowly (deploymen$\mathrm{t}$ mode), the amplitude of the oscillation becomes smaller. On the other hand, if the tether is shortened (retrieval mode), the oscillation becomes larger. This observation coincides with the assertion that the dynamics is stable during the deployment mode, but unstable during the retrieval mode.
\end{abstract}

Keywords: Tethered-Satellite system, Adiabatic invariance, Stability, Elliptic integral, Action variable.

\section{Introduction}

A joint mission of USA and Italy, called TetheredSatellite-System (TSS), has been operated for a few years. The idea of using tether in space has been discussed for many years, such as the $s k y$-hook discussed in [8]. The TSS consists of a massive satellite (such as space shuttle) and a subsatellite connected by a long tether. The operational mode can be divided into three phases. In station-keeping phase, the system is kept in an earth-pointing attitude moving around the earth, so that some scientific experiments can be conducted. Before the station-keeping phase, the payload must be deployed from the shuttle by first ejected from the shuttle cargo bay, and then the tether is released with a reel mechanism. This is the so-called deployment phase. On the other hand, the payload may need to be retrieved back after the experiment is done. During this retrieval mode, the length of the tether must be shortened smoothly by also a reel mechanism. It is noted that during both the deployment and retrieval modes, the length of the tether is changed slowly. One effect of the slowly-varying length can be observed from the theory of adiabatic invariance.

After the Lorentz pendulum problem was raised in the 1911 Solvay Conference, cf.[14], the notion of adiabatic invariance has attracted the attention of many researchers in classical mechanics and quantum mechanics. It was proved that if the length of the pendulum is changed slowly, the energy is proportional to the frequency with an almost constant ratio, the so-called adiabatic invariance. This quantity may correspond to some quantization of state which is unchanged during slow variations of the atomic environment in quantum mechanics. Adiabatic invariances for many physical systems have been computed, cf.[7], [6],[11]. For a Hamiltonian system, it has been proved that the action variable is indeed an adiabatic invariance under suitable conditions, cf.[4], [9]. This paper thus attempts to find the adiabatic invariance for some approximate model of TSS during deployment/retrieval modes by computing the corresponding action variable. With this invariance, some physical insight for the dynamics of TSS can be gained.

A profound, historical review on the dynamics and control of TSS can be found in [1]. Most literature use linear analysis or numerical calculations to attack the problem in deployment/retrieval phase. Some work study the nonlinear phenomenon of TSS and proved that under certain conditions, the Earth-pointing stationkeeping mode is stable, cf.[16]. On the other hand, by some linearization, it is observed that the dynamics of retrieval mode is intrinsically unstable, cf.[1]. It is our goal here to study the problem from another point of view. From the adiabatic invariance we computed, 
it is observed that the length of the tether is inverse proportional to the amplitude of the oscillation. This means that as the tether length being shortened, the amplitude of oscillation becomes larger, which somewhat elucidates the instability of the motion in the retrieval mode. The conclusion we made coincides with the results before.

In what follows, Section 2 describes the physical model of TSS which is adopted for analysis. The notion of adiabatic invariance and the computation for our model are discussed in Section 3 . Some concluding remarks are given in Section 4.

\section{System Descriptions}

The TSS can be modeled as two point masses $m_{1}, m_{2}$ connected by a taut rod-like tether, cf. Fig.1. Although this model seems to be oversimplified, it may give some insight on the dynamical behaviors. We are interested in the motion of the system during deployment phase and retrieval phase. It has been observed that the system may act as a dumbbell oscillating about the Earth pointing direction, cf.[2],[13]. It is assumed that the length of the tether $l$ varies slowly enough during the motion, such that the tether length $l=l(\epsilon t), \epsilon \ll 1$, is almost a constant within one period of the oscillation. On the other hand, the variation of the tether length is smooth such that the condition for applying the theory of adiabatic invariance is satisfied, similar to the Lorentz pendulum problem, cf. [4].

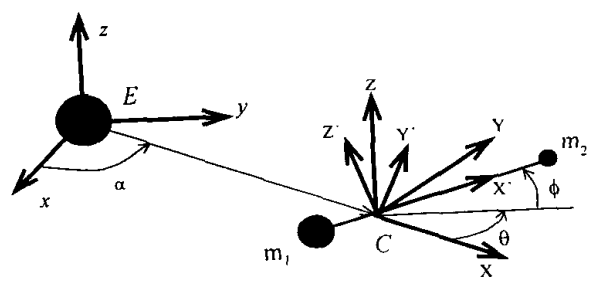

Figure 1: type I motion

Let $E$ denote the position of the center of the Earth, and $C$ denote the position of the center of mass of TSS. There are two types of motion we shall consider in the following analysis. For type I, cf. Fig.1, the center of mass moves around the Earth in circular orbit, satisfying the Kepler's law. For the motion of type II, cf. Fig.2, it is assumed that the position of $m_{1}$ moves around the Earth in circular orbit. Since typically $m_{1}$ represents the space shuttle, which has appropriate control on its orbit, type II motion may be more realistic.

To describe the kinematics, an inertial frame $\{x, y, z\}$ is affiliated to $\mathrm{E}$, with $z$ perpendicular to the orbital plane; and an orbital frame $\{X, Y, Z\}$ is constructed, with $X$ being parallel to the radius vector of the orbit, $Z$ being perpendicular to the orbital plane, and $Y$ completing the triad. Moreover, we affiliate a

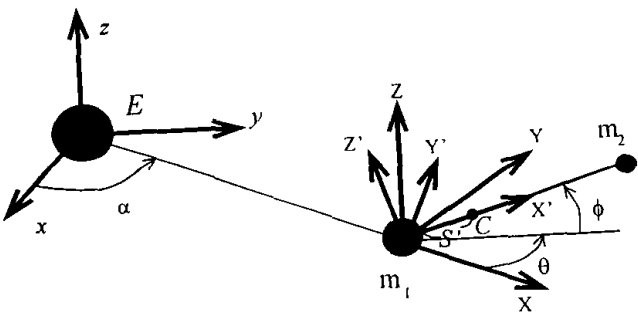

Figure 2: type II motion

body frame $\left\{X^{\prime}, Y^{\prime}, Z^{\prime}\right\}$ to TSS such that $X^{\prime}$ being parallel to the direction of the tether. Let $\theta, \phi$ denote the azimuth and elevation angle of $X^{\prime}$ relative to the orbital frame, respectively. cf. Fig. 1 or 2 . In some literature, $\mathrm{cf} .[1],[2], \theta$ is called the in-plane angle and $\phi$ is named the out-of-plane angle. Accordingly, the coordinate transformation from the orbital frame to the inertial frame can be expressed as

$$
\begin{aligned}
A & =\operatorname{Rot}(3, \alpha) \\
& =\left[\begin{array}{ccc}
\cos \alpha & -\sin \alpha & 0 \\
\sin \alpha & \cos \alpha & 0 \\
0 & 0 & 1
\end{array}\right]
\end{aligned}
$$

where $\alpha$ denotes the true anomaly. The coordinate transformation from the body frame to the orbital frame is

$$
\begin{aligned}
B & =\operatorname{Rot}(3, \theta) \operatorname{Rot}(2,-\phi) \\
& =\left[\begin{array}{ccc}
\cos \theta & -\sin \theta & 0 \\
\sin \theta & \cos \theta & 0 \\
0 & 0 & 1
\end{array}\right]\left[\begin{array}{ccc}
\cos \phi & 0 & -\sin \phi \\
0 & 1 & 0 \\
\sin \phi & 0 & \cos \phi
\end{array}\right] .
\end{aligned}
$$

The above matrices $\operatorname{Rot}(\cdot, \cdot)$ are $3 \times 3$ rotation matrices which form the special orthogonal group $S O(3)$. For any element $D \in S O(3)$, which may represent a coordinate transformation. We have $D^{T} D=1$ and $\operatorname{det} D=1$, where 1 denotes the $3 \times 3$ identity matrix. Let the operator - denote the natural isomorphism between $\mathbb{R}^{3}$ and $s o(3)$, the space of $3 \times 3$ skew-symmetric matrices, defined by

$$
\left(\begin{array}{l}
w_{1} \\
w_{2} \\
w_{3}
\end{array}\right) \equiv\left(\begin{array}{ccc}
0 & -w_{3} & w_{2} \\
w_{3} & 0 & -w_{1} \\
-w_{2} & w_{1} & 0
\end{array}\right)
$$

By taking the time derivative on both side of $D^{T} D=1$, the kinematic relationship

$$
\dot{D}=D \hat{\Gamma}
$$

can be obtained, where (in mechanics) the vector $\Gamma$ corresponds to the instantaneous angular velocity.

For the transformation matrices defined in (1) and (2), we have

$$
\dot{A}=A \hat{\Omega}, \quad \dot{B}=B \hat{\Theta},
$$


where

$\Omega=\left(\begin{array}{c}0 \\ 0 \\ \dot{\alpha}\end{array}\right)_{X-Y-Z}, \quad \Theta=\left(\begin{array}{c}\dot{\theta} \sin \phi \\ -\dot{\phi} \\ \dot{\theta} \cos \phi\end{array}\right)_{X^{\prime}-Y^{\prime}-Z^{\prime}}$.

Let $\mathbf{r}_{g}, \mathbf{r}_{c}$ denote the center of gravity and the center of mass of the system, respectively. It has been shown, cf. [15] that the orbital angular velocity, $\omega=\dot{\alpha}$, can be computed from

$$
\mu=\omega^{2}\left|\mathbf{r}_{g}\right|<\mathbf{r}_{g}, \mathbf{r}_{c}>
$$

where $|\cdot|$ denotes the norm of vector; $\langle\cdot, \cdot\rangle$ represents the inner product of two vectors, and $\mu$ is the gravitational constant.

We are now ready to describe the kinematics of the system. Let $\mathbf{R}_{0}$ denote the radius vector of the orbit observed from orbital frame $\{X, Y, Z\}$ and $R_{0}$ be its magnitude. The position vector of an element of TSS expressed in inertial frame can then be written as

$$
\begin{aligned}
\mathbf{r} & =A\left(\mathbf{R}_{0}+B \mathbf{R}^{\prime}\right) \\
& =A \mathbf{R}_{0}+C \mathbf{R}^{\prime},
\end{aligned}
$$

where $\mathbf{R}^{\prime}$ denotes the position of that element relative to the body frame, and $C=A B$. Taking time derivative on both side of (5) yields

$$
\dot{\mathbf{r}}=A \hat{\Omega} \mathbf{R}_{0}+C \hat{\Lambda} \mathbf{R}^{\prime}+A B \dot{\mathbf{R}}^{\prime},
$$

where $\hat{\Lambda}$ can be found to be equal to $B^{T} \hat{\Omega} B+\hat{\Theta}$. Note that $\left|\dot{\mathbf{R}}^{\prime}\right|$ is proportional to the tether length rate $\dot{l}$. Since $l$ is assumed to vary slowly, $l$ can be regarded almost unchanged during a period of the oscillating motion of TSS. Thus, $A B \mathbf{R}^{\prime}$ is much smaller than the other terms of (6), and could be neglected.

The kinetic energy of the system can be obtained from

$$
T=\frac{1}{2} \int_{m_{1}+m_{2}+m_{t}}<\dot{\mathbf{r}}, \dot{\mathbf{r}}>d m
$$

where $m_{t}$ denotes the mass of the tether in taut position with length $l$. If the total length of the tether is $L_{0}$ and $\rho$ denotes the density (mass/length) of the tether, then

$$
m_{1}=m_{10}+\rho\left(L_{0}-l\right), m_{t}=\rho l,
$$

where $m_{10}$ is the mass of the main structure with the tether excluded. Therefore, the total mass $M=m_{1}+$ $m_{2}+m_{t}=m_{10}+m_{2}+\rho L_{0}$ is invariant with time. Neglecting the term $A B \mathbf{R}^{\prime}$, the kinetic energy can be obtained by substituting eq. (6) into (7) as

$$
\begin{gathered}
T=\frac{1}{2} \int_{M}\left(<A \hat{\Omega} \mathbf{R}_{0}, A \hat{\Omega} \mathbf{R}_{0}>+2<A \hat{\Omega} \mathbf{R}_{0}\right. \\
\left.C \hat{\Lambda} \mathbf{R}^{\prime}>+<C \hat{\Lambda} \mathbf{R}^{\prime}, C \hat{\Lambda} \mathbf{R}^{\prime}>\right) d m
\end{gathered}
$$

By using $A^{T} A=1, D^{T} D=1$, the first term and the third term of (8) be collected as

$$
\frac{1}{2}\left(M|\Omega|^{2}\left|\mathbf{R}_{0}\right|^{2}+\Lambda^{T} \mathbf{I} \Lambda\right)
$$

where $\mathbf{I}=\int_{M}\left(<\mathbf{R}^{\prime}, \mathbf{R}^{\prime}>1-\mathbf{R}^{\prime} \mathbf{R}^{\prime} T\right) d m$ is the inertia matrix. It is noted that for type I motion, this inertia matrix is relative to the center of mass $\mathrm{C}$. However, for type II motion, the inertia matrix must be computed with respect to the point $m_{1}$. The second term of (8) needs further treatment. For type I motion, it is observed that

$$
\int_{M} \mathbf{R}^{\prime} d m=\mathbf{0}
$$

thus the kinetic energy is simply (9). However, for type II motion, we define $\mathbf{R}^{\prime}=\mathbf{S}^{\prime}+\mathbf{R}_{r e l}^{\prime}$, where $\mathbf{S}^{\prime}$ denotes the vector from $m_{1}$ to $C$; and $\mathbf{R}_{r e l}^{\prime}$ denotes the vector from $C$ to the element on TSS. Noting that $\int_{M} R_{r e l}^{\prime} d m=0$, and substituting the expression of I, $B, \hat{\Omega}, \hat{\Lambda}$ in terms of moment of inertia $I$, and $\theta, \phi, \dot{\theta}, \dot{\phi}, \omega$, into (9), the kinetic energy (7) has an explicit form as

$$
\begin{aligned}
T= & \frac{1}{2} M R_{0}^{2} \omega^{2}+\frac{1}{2} I\left(\dot{\phi}^{2}+(\omega+\dot{\theta})^{2} \cos ^{2} \phi\right)+M R_{0} \\
& \omega(-\sin \theta \sin \phi \dot{\phi}+\cos \theta \cos \phi(\omega+\dot{\theta})) S^{\prime}, \quad(10)
\end{aligned}
$$

where $S^{\prime}=\left|\mathbf{S}^{\prime}\right|$. It is observed that by letting $S^{\prime}=0$, $T$ becomes the kinetic energy for type I motion.

The potential energy of TSS can be calculated from

$$
\begin{aligned}
V= & \frac{-\mu m_{1}}{\left|\mathbf{R}_{0}+B \mathbf{R}_{1}^{\prime}\right|}+\frac{-\mu m_{2}}{\left|\mathbf{R}_{0}+B \mathbf{R}_{2}^{\prime}\right|}+ \\
& \int_{R_{1}^{\prime}}^{R_{2}^{\prime}} \sqrt{\left|\mathbf{R}_{0}+B \mathbf{R}^{\prime}\right|} d R^{\prime},
\end{aligned}
$$

in which, $\left(\left|\mathbf{R}_{0}+B \mathbf{R}^{\prime}\right|\right)^{-1}$ is equal to

$$
\left(R_{0}^{2}+2 \cos \theta \cos \phi R_{0} R^{\prime}+R^{\prime 2}\right)^{-\frac{1}{2}} \text {. }
$$

Letting $\delta=\frac{R^{\prime}}{R_{0}},(12)$ can be expanded as a series of $\delta^{n}, n=1,2,3 \ldots$, i.e.

$$
\begin{aligned}
& \frac{1}{R_{0}}\left(1-\cos \theta \cos \phi \delta+\frac{1}{2}\left(3 \cos ^{2} \theta \cos ^{2} \phi-1\right) \delta^{2}\right. \\
& \text { +higher order terms }) .
\end{aligned}
$$

Since $\delta \ll 1$, the higher order terms can be neglected. Thus, (11) can be reduced to

$$
\begin{aligned}
V= & -\frac{\mu M}{R_{0}}+\frac{\mu M S^{\prime}}{R_{0}^{2}} \cos \theta \cos \phi \\
& -\frac{\mu I}{2 R_{0}^{3}}\left(3 \cos ^{2} \theta \cos ^{2} \phi-1\right),
\end{aligned}
$$


where $S^{\prime}=0$ corresponds to the type I motion. Finally, the Lagrangian $L=T-V$ can be written as

$$
\begin{aligned}
L= & C_{1}+\frac{1}{2} I\left(\dot{\phi}^{2}+(\omega+\dot{\theta})^{2} \cos ^{2} \phi\right)+M R_{0} S^{\prime} \\
& \left(-\sin \theta \sin \phi \omega \dot{\phi}+\cos \theta \cos \phi\left(\omega^{2}-\frac{\mu}{R_{0}^{3}}\right.\right. \\
& +\omega \dot{\theta}))+\frac{\mu I}{2 R_{0}^{3}}\left(3 \cos ^{2} \theta \cos ^{2} \phi-1\right)
\end{aligned}
$$

where $C_{1}=\frac{1}{2} M R_{0}^{2} \omega^{2}+\frac{\mu M}{R_{0}}$ is independent of $\theta, \phi, \dot{\theta}, \dot{\phi}$. By Legendre transformation, the Lagrangian $L$ can be transformed into a Hamiltonian, so that the dynamics becomes a Hamiltonian system.

\section{The Adiabatic Invariance}

Formally, an adiabatic invariance is defined by the following statement,

Definition. a function $J(p, q, l)$ is called an adiabatic invariance if for every $\kappa>0$ there is an $\epsilon_{0}=\epsilon_{0}(\kappa)$ such that for $\epsilon<\epsilon_{0}$ the variation of $J(p(t), q(t), l(\epsilon t))$ for $0 \leq t \leq \frac{1}{\epsilon}$ does not exceed $\kappa$,

where $(p, q)$ are the canonical variables in Hamiltonian formulations; $l=l(\epsilon t), \epsilon \ll 1$ is a slowly varied parameter of the system, cf.[3],[4].

For a Hamiltonian system, such as the one described above, it has been proved that the action variable

$$
J=\oint p d q
$$

where $q, p$ denote the generalized coordinate and the generalized momentum variable, respectively, is an adiabatic invariance. Accordingly, we compute the action variable for our model discussed in Section 2. To make the computations tractable, the in-plane motion and out-of-plane motion are considered.

\subsection{In-plane motion}

For in-plane motion of TSS, it is assumed that $\phi=\dot{\phi}=$ 0 . Therefore the Lagrangian $L$ can be simplified as

$$
\begin{aligned}
L_{\theta}= & C_{1}+\frac{1}{2} I(\omega+\dot{\theta})^{2}+M R_{0} S^{\prime} \cos \theta \\
& \left(\omega^{2}-\frac{\mu}{R_{0}^{3}}+\omega \dot{\theta}\right)+\frac{\mu I}{2 R_{0}^{3}}\left(3 \cos ^{2} \theta-1\right)(16)
\end{aligned}
$$

Applying Legendre transformation, the Hamiltonian can be found to be

$$
H_{\theta}=\frac{1}{2 I}\left(P_{\theta}-F_{1}\right)^{2}-\omega\left(P_{\theta}-F_{1}\right)-F_{2},
$$

where $P_{\theta}=\frac{\partial L_{I}}{\partial \dot{\theta}}=I(\omega+\dot{\theta})+F_{1}$ denote the momentum variable, and ${ }^{\partial}$

$$
\begin{aligned}
& F_{1}=M R_{0} S^{\prime} \omega \cos \theta \\
& F_{2}=\frac{F_{1}}{\omega}\left(\omega^{2}-\frac{\mu}{R_{0}^{3}}\right)+\frac{\mu I}{2 R_{0}^{3}}\left(3 \cos ^{2} \theta-1\right)+C_{1} .
\end{aligned}
$$

The action variable $J_{\theta}$, that is, the adiabatic invariance, can be obtained by calculating $\oint P_{\theta} d \theta$, cf.[9], [4]. It can be shown that

$$
P_{\theta}=F_{1}+I \omega \pm I \sqrt{\omega^{2}+\frac{2}{I}\left(F_{2}+H_{\theta}\right)}
$$

Since $F_{1}, F_{2}$ both are even functions of $\theta$, and noting that $H_{\theta}, \omega$ and $I$ are almost invariant within one period of in-plane motion, $J_{\theta}$ can be reduced to

$$
J_{\theta}=4 \sqrt{\frac{3 \mu}{R_{0}^{3}}} I \int_{0}^{\theta_{\max }} \sqrt{\cos ^{2} \theta-a \cos \theta-b} d \theta
$$

where

$$
\begin{aligned}
& a=\frac{\left(\frac{\mu}{R_{0}^{3}}-\omega^{2}\right) R_{0} M S^{\prime}}{\frac{3 \mu I}{2 R_{0}^{3}}} \\
& b=\frac{\frac{\mu I}{2 R_{0}^{3}}-\frac{I \omega^{2}}{2}-H_{\theta}-C_{1}}{\frac{3 \mu I}{2 R_{0}^{3}}}
\end{aligned}
$$

and $\theta_{\max }=\arccos \left(\frac{a}{2}+\sqrt{b+\frac{a^{2}}{4}}\right)$ is the amplitude of in-plane oscillation. To assure that TSS undergoes a periodic libration in orbit, it is required that $\frac{a}{2}+\sqrt{b+\frac{a^{2}}{4}} \leq 1$ and $b+\frac{a^{2}}{4}>0$, and from which, $a+b \leq 1$ is obtained. For type I motion, $S^{\prime}=0$, and thus $a=0$. On the other hand, for type II motion, it is observed from (4) that $a>0$, since $R_{0}<r_{c}$, and $R_{0}<r_{g}$. Moreover, the libration motion is assured by the condition $b>0$, which is also assumed in the following discussion.

For type I motion, $a=0$, and the integral in $J_{\theta}$ can be expressed in terms of complete elliptic integrals, cf.[10], [5], such that

$J_{\theta}^{\mathrm{I}}=4 \sqrt{\frac{3 \mu}{R_{0}^{3}}} I \sin ^{2} \theta_{\max }\left[\mathcal{K}\left(\sin \theta_{\max }\right)-\mathcal{D}\left(\sin \theta_{\max }\right)\right]$.

It is noted that if $J_{\theta}^{\mathrm{I}}$ is a constant, the moment of inertia $I$ has certain relationship with the amplitude of oscillation $\theta_{\max }$. To gain further insight on their relation, a physical example is considered, with $m_{10}=$ $80000 \mathrm{~kg}, m_{2}=500 \mathrm{~kg}, \rho=8.2 \mathrm{~kg} / \mathrm{km}, L_{0}=$ $100 \mathrm{~km}, R_{0}=r_{c}=6800 \mathrm{~km}$. Fig. 3 plots the relation between $I$ and the length of tether $l$, while Fig.4 plots the relation between $\sin ^{2} \theta_{\max }(\mathcal{K}-\mathcal{D})$ and $\theta_{\max }$. Thus, if $l$ is varied slowly, we may find the corresponding $I$ from Fig. 3 , and then compute $\sin ^{2} \theta_{\max }(\mathcal{K}-\mathcal{D})$ by the constancy of the adiabatic invariance. With the value of $\sin ^{2} \theta_{\max }\left[\mathcal{K}\left(\sin \theta_{\max }\right)-\mathcal{D}\left(\sin \theta_{\max }\right)\right]$, the amplitude of oscillation $\theta_{\max }$ can then be obtained from Fig.4. As an example, if a deviation $\theta=4^{\circ}$ occurs when $l=50 \mathrm{~km}$, and the tether is enlongated to $70 \mathrm{~km}$, then 
the oscillation becomes $\theta_{\max } \approx 2.74^{\circ}$. On the other hand, if the tether is shortened to $30 \mathrm{~km}$, the oscillation is then $6.98^{\circ}$. From the above discussion, we conclude that for type I in-plane motion, the length of tether is inversely varying with respect to the amplitude of oscillation.

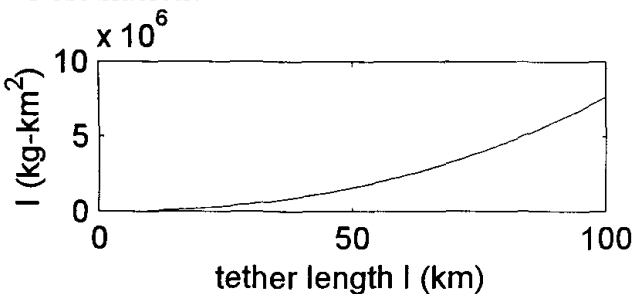

Figure 3: $I$ vs. $l$

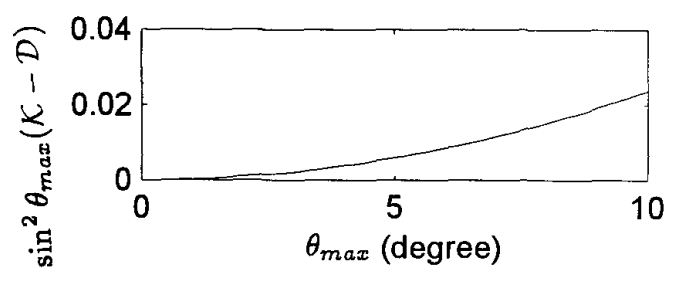

Figure 4: $\sin ^{2} \theta_{\max }(\mathcal{K}-\mathcal{D})$ vs. $\theta_{\max }$

For type II motion, the integral in (18) is a general elliptic integral, cf.[12], [5], which can be dealt with by applying a series of suitable transformations. However, the integral expressions include a lengthy algebraic function with $a, b$ as the parameters, which makes the calculation intractable. Then, numerical computations are adopted in order to gain some physical insight. Let (18) be expressed as

$$
\begin{aligned}
J_{\theta}^{I I}= & 4 \sqrt{\frac{3 \mu}{R_{0}^{3}}} I \int_{0}^{\theta_{\max }} \sqrt{\left(\cos \theta-\cos \theta_{\max }\right)} \\
& \sqrt{\left(\cos \theta-\left(a-\cos \theta_{\max }\right)\right)} d \theta \\
= & 4 \sqrt{\frac{3 \mu}{R_{0}^{3}}} I F_{3}\left(\theta_{\max }, a\right) .
\end{aligned}
$$

It is noted that $I, a$ are changed as the tether length being varying, which gives rise to the variation of $\theta_{\max }$ for keeping $J_{\theta}^{I I}$ being an adiabatic invariance. Consider a physical example, $m_{10}=2000 \mathrm{~kg}, m_{2}=500 \mathrm{~kg}, \rho=$ $8.2 \mathrm{~kg} / \mathrm{km}, R_{0}=r_{1}=6800 \mathrm{~km}, L_{0}=100 \mathrm{~km}$. The relations between $I, a$ and $l$ can be found in Fig.6, while the relation between $F_{3}$ and $\theta_{m a x}, a$ is plotted in Fig.5. When a deviation $\theta_{\max }=4^{\circ}$ occurs at $l=40 \mathrm{~km}$, we have $a=0.4331, I=9.7493 \times 10^{5} \mathrm{~kg}-$ $\mathrm{km}^{2}, F_{3}\left(\theta_{\text {max }}, a\right)=0.0034$. If $l$ is slowly extended to $60 \mathrm{~km}$, we find $a=0.5002, I=23.904 \times 10^{5} \mathrm{~kg}-\mathrm{km}^{2}$. To keep $J_{\theta}^{I I}$ being a constant, it is required that $F_{3}\left(\theta_{\max }, a\right)=0.0013866$. Thus, the oscillation $\theta_{\max }$ can be read out from Fig. 5 as $\theta_{\max } \approx 2.589^{\circ}$. On the other hand, if the tether length is shortened slowly to $20 \mathrm{~km}$, it can be checked that $\theta_{\max }$ becomes $8.335^{\circ}$, by applying similar process. This numerical computation also shows that for type II in-plane motion, the tether length is inversely varying with respect to the amplitude of oscillation.

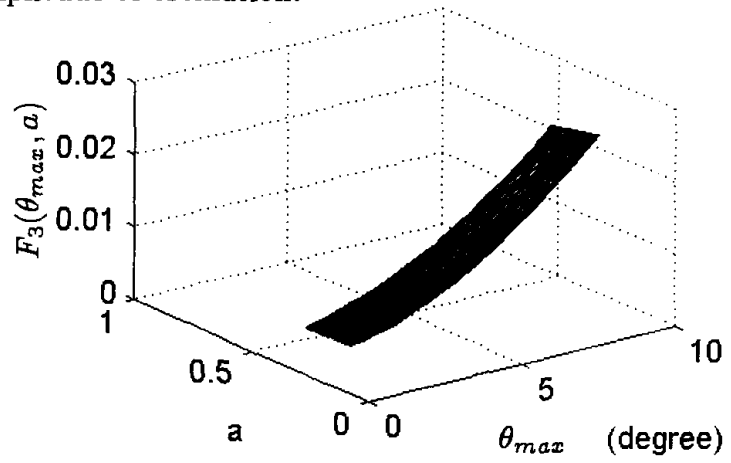

Figure 5: $F_{3}\left(\theta_{\max }, a\right)$ vs. $a$ and $\theta_{\max }$

As discussed in [1], a linearized dynamical equation

$$
\theta^{\prime \prime}+2\left(l^{\prime} / l\right) \theta^{\prime}+3 \theta=-2\left(l^{\prime} / l\right)
$$

can be used as a model for deployment/retrieval control system. It can then be concluded that if the length change rate $l^{\prime}$ is positive/negative then the motion in $\theta$ direction is stable/unstable. Because $l$ is positive/negative corresponding to the tether in deployment/retrieval process, the results we obtained above coincide with the observation made before.
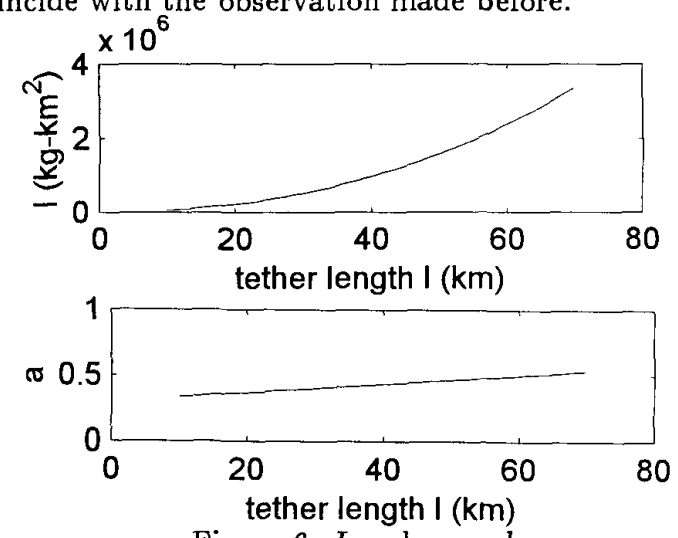

Figure 6: $I$ and $a$ vs. $l$

\subsection{Out-of-plane motion}

Now we consider the motion of TSS with $\theta=0$, the so-called out-of-plane motion. By applying the steps analogous to Section 3.1, the adiabatic invariances for type I motion and type II motion can be expressed as

$$
\begin{aligned}
J_{\phi}^{I} & =4 \sqrt{\omega^{2}+\frac{3 \mu}{R_{0}^{3}}} I \sin ^{2} \phi_{\max }[\mathcal{K}-\mathcal{D}], \\
J_{\phi}^{I I} & =4 \sqrt{\omega^{2}+\frac{3 \mu}{R_{0}^{3}}} I F_{3}\left(\phi_{\max }, c\right),
\end{aligned}
$$

respectively; where $\phi_{\max }, c$ have similar characters as $\theta_{\max }, a$ defined in Section 3.1. Comparing the above 
expression with (19), (20), the only difference lies in the pre-integral multiplier, i.e. $\sqrt{\omega^{2}+\frac{3 \mu}{R_{0}^{3}}}$. Recalling that $\omega$ is affected by the varied tether length, if the two examples in Section 3.1 are reconsidered, the variations for $\sqrt{\omega^{2}+\frac{3 \mu}{R_{0}^{3}}}$ vs. $l$ are plotted in Fig.7 and Fig.8, respectively. It is observed that for different tether lengths, $\sqrt{\omega^{2}+\frac{3 \mu}{R_{0}^{3}}}$ changes not significantly. Therefore, the results for these examples in out-of-plane oscillations would be almost the same as that for in-plane motions discussed in Section 3.1.

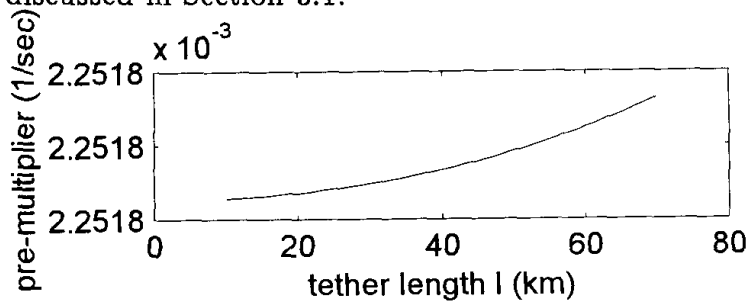

Figure $7: \sqrt{\omega^{2}+\frac{3 \mu}{R_{0}^{3}}}$ vs. $l$ for type I motion

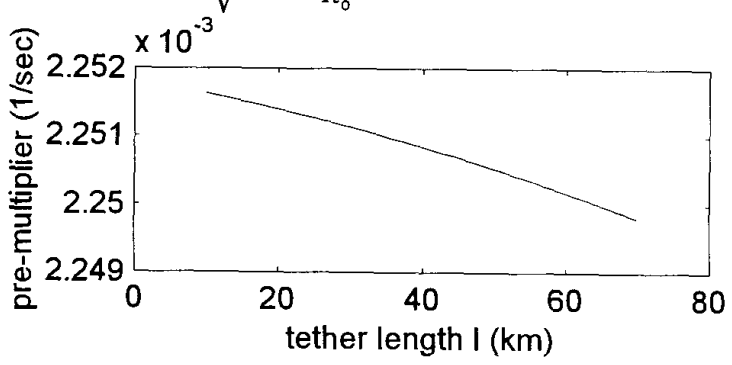

Figure $8: \sqrt{\omega^{2}+\frac{3 \mu}{R_{0}^{3}}}$ vs. $l$ for type II motion

\section{Conclusion}

From the above analysis, an adiabatic invariance was derived for the motion of TSS in the deploymen$t /$ retrieval phases. For both the in-plane motion and the out-of-plane motion, it is observed that the amplitude of oscillation and the length of the tether vary inversely. Consequently, as the tether being pulled back during the retrieval phase, the oscillation becomes larger, which may induce instability to the system. Although the analysis is based on a simplified model of TSS and the in-plane motion and the out-of-plane motion are considered separately, the conclusion we made has given some insight on the dynamical behavior. We note that the action variable is in fact the order one adiabatic invariance, the other adiabatic invariance of different orders may be pursuit in the future.

\section{References}

[1] A.K.Misra and V.J.Modi. A Survey on the Dynamics and Control of Tethered Satellite. In Teth- er in Space, pages 667-719. American Astronautical Society, 1986.

[2] David A. Arnold. The behavior of long tethers in space. The Journal of the Astronautical Sciences, $35(1): 3-18,1987$.

[3] V.I. Arnold, editor. Dynamical Systems III, volume 3 of Encyclopaedia of Mathematical Sciences. Springer-Verlag, 1988.

[4] V.I. Arnold. Mathematical Methods of Classical Mechanics. Springer-Verlag, second edition, 1989.

[5] Authur Cayley. Elementary Treatise on Elliptic Functions. Dover, 1961.

[6] W.A. Day. Adiabatic Invariants for Strings, Membranes, and Beams of Slowly-Varying Dimensions. Quarterly of Applied Mathematics, 45(2):389-398, 1987.

[7] Djordje S. Djukic. Adiabatic Invariants for the Nonconservative Kepler's Problem. Celestial Mechanics and Dynamical Astronomy, 56:523-540, 1993.

[8] G.Colombo et. al. Shuttle-Borne 'Skyhook' : A New Tool for Low-Orbital-Altitude Research. Smithsonian Institution Astrophysical Observatory, September 1974.

[9] H. Goldstein. Classical Mechanics. AddisonWesley Publishing Company, second edition, 1980.

[10] Eugene Jahnke and Fritz Emde. Tables of Functions With Formulae and Curves. Dover, fourth edition, 1945.

[11] Martin Kruskal. Asymptotic Theory of Hamiltonian and other Systems with all Solutions Nearly Periodic. Journal of Mathematical Physics, 3(4):806$828,1962$.

[12] Derek F. Lawden. Elliptic Functions and Applications. Springer-Verlag, 1989.

[13] E.M. Levin. Nearly-uniform deployment strategy for space tether system. Acta Astronautica, 32(5):399-403, 1994.

[14] J.E. Littlewood. Lorentz's Pendulum Problem. Annals of Physics, 21:232-242, 1963.

[15] L.S. Wang and S.F. Cheng. Dynamics of Springconnected Massive in Orbit, to appear in Celestial Mechanics and Dynamical Astronomy, 1996.

[16] L.S. Wang, S.J. Chern, and C.W. Shih. On the Dynamics of a Tethered Satellite System. Arch. Rational. Mech. Anal., 127:297-318, 1994. 